East African Medical Journal Vol. 86 No. 5 May 2009

LYMPHOMAS DIAGNOSED IN UGANDA DURING THE HIV / AIDS PANDEMIC

S. Kalungi, ' MBChB, MMed, Section for Pathology, The Gade Institute, University of Bergen, Bergen, Norway and Department of Pathology, Makerere University College of Health Sciences, P. O. Box 7072, Kampala, Uganda, H. Wabinga, MD, Department of Pathology, Makerere University College of Health Sciences, P. O. Box 7072, Kampala, Uganda, A. Molven, PhD and L. Bostad, MD, Section for Pathology, the Gade Institute, University of Bergen, Bergen, Norway and Department of Pathology, Haukeland University Hospital, Bergen, Norway

Request for reprints to: Dr. S. Kalungi, Makerere University College of Health Sciences, P. O. Box 7072, Kampala, Uganda

\title{
LYMPHOMAS DIAGNOSED IN UGANDA DURING THE HIV/AIDS PANDEMIC
}

\author{
S. KALUNGI,'H. WABINGA, A. MOLVEN and L. BOSTAD
}

\begin{abstract}
Background: There are numerous reports from different countries documenting a change in frequency and profile of lymphomas after the onset of the HIV/AIDS pandemic. In Uganda little is known concerning the distribution of lymphoma subtypes diagnosed at the Department of Pathology, Makerere University College of Health Sciences during this period.

Objective: To examine the frequency and diagnostic profile of lymphomas diagnosed in Uganda in the HIV/AIDS era.

Design: Retrospective study.

Setting: Department of Pathology, Makerere University College of Health Sciences, Kampala, Uganda.

Subjects: One thousand and thirteen patients diagnosed with lymphomas in the period 1980-1989.

Results: The most common type of non-Hodgkin lymphoma was Burkitt lymphoma $(36 \%)$. The frequencies of lymphocytic and histiocytic types were $34.5 \%$ and $8.2 \%$ respectively.

Conclusion: There was a decrease in histopathologically diagnosed lymphomas in Uganda in the period 1980-1989. Burkitt lymphoma continues to be the most common subtype diagnosed, some major lymphoma subtypes like T-cell and follicular lymphomas were not reported in the country in the HIV/AIDS era.
\end{abstract}

\section{INTRODUCTION}

Lymphomas have been found to increase during the HIV / AIDS pandemic in most populations. In the United States theincrease of non-Hodgkin lymphoma (NHL) has been estimated to be 100-fold in the population of HIV-positive patients. Most of these cases are in the high-grade group. A nearly 10 -fold increased risk of Hodgkin lymphoma (HL) and a nearly 60 -fold increased risk of NHL have been found among HIV-positive individuals in Europe $(1,2)$.

In Africa, there are conflicting reports on the relationship between lymphomas and HIV/ AIDS with some reports indicating an increase in incidence whereas some studies showed no increase in lymphoma cases among HIV/AIDS patients. An autopsy series from the Ivory Coast did not provide any evidence for an increase of frequency of childhood lymphoma (3). However, data from population-based cancer registries throughout Africa indicate an increase in the incidence of NHL. A study by Wabinga et al (4) found a two-fold, statistically significant increase in NHL in Kyadondo County in Uganda. The HIV / AIDS prevalence in Uganda was reported to be about 30\% in the 1980-1989 period (5). This high prevalence of HIV would be expected to have had an impact on the pattern of lymphomas in Uganda.

The distribution of different lymphoma subtypes as investigated using pathology laboratory-based data was last published in 1973 (6). Most of the studies carried out in Uganda have been from a populationbased cancer registry and often does not include lymphoma subtypes $(4,5,7,8)$. Our study is based exclusively on the diagnosis as found in the histology reports "pathology based data" retrieved from the files of the "Department of pathology".

The updated WHO classification of lymphomas includes integration of morphological, immunophenotypical and molecular methods. The 
standard examination method used in our laboratory, however, is light microscopy of haematoxylin and eosin (H\&E) stained sections. The purpose was to examine the profile of lymphomas diagnosed in the HIV / AIDS era. We also compared our data to a similar study carried out in the 1960s (6). Our findings highlight trends in the occurrence of diagnosed lymphoma subtypes and reveal the current state of lymphoma histopathology in Uganda.

\section{MATERIALS AND METHODS}

Sampling method: Consecutive, purposive sampling was used.

Design: Retrospective study.

Rationale for themethod: The Department of Pathology, Makerere University College of Health Sciences has been the sole provider of diagnostic histopathology for the entire country of Uganda up to 1990. The population of Uganda was estimated to be 16.7 million according to the 1990 Uganda population and housing census as compared to 9.5 million in 1969. $\mathrm{H} \& \mathrm{E}$ staining remains the only stain used for routine diagnosis in the Department of Pathology, as was the case in the 1960s. The same method of review of histology records is acceptable and used for most of the data originating from population-based cancer registries. Histopathologic examination and diagnosis also form the basis of patient treatment.

Procedure: Records of cases of lymphoma diagnosed in the years 1980-1989 and coded as ICD-10 codes 200, 201, 202 or C81-C85 were retrieved from the files of the Department between January and September 2005. Data including age, site of biopsy and histological diagnosis were obtained from the request forms. The NHL were categorised as low or high grade according to the criteria from the Kiel classification.

Setting: Department of Pathology, Makerere University College of Health Sciences, Kampala, Uganda.

Inclusion criteria: All recorded cases of lymphoma between 1980-1989.

Exclusion criteria: Leukaemia and cases without specified type.

Data handling: The forms with the required data were retrieved from the archives; data registered on coded forms, and then computerised. Analysis was done using the Epi-info software package.

\section{RESULTS}

A total of 1013 patients were recorded with a diagnosis of lymphoma in the period 1980-1989. Eight hundred and thirty nine cases with the histological type specified were included in further analysis. Gender was specified in 714 cases. Male preponderance was observed in the majority of lymphoma subtypes (Table 1). The results were compared to the data of D.H.Wright from 1964-1968 (6). There were 619 (82.5\%) non Hodgkin lymphoma (NHL) cases in 1964-1968 compared to $740(88.2 \%)$ in 1980-1989 and 128 (17.5\%) Hodgkin lymphoma (HL) cases in 1964-1968 compared to $99(11.8 \%)$ in 1980-1989. NHL were 7.5 times more common than HL in 1980-1989 compared to 4.8 times in the preHIV / AIDS period.

Table 1

Gender and distribution of Hodgkin and non-Hodgkin's lymphomas diagnosed at the Department of Pathology, Makerere University College of Health Sciences in 1980-1989

\begin{tabular}{|c|c|c|c|}
\hline \multirow{2}{*}{$\begin{array}{l}\text { Lymphoma } \\
\text { type }\end{array}$} & \multicolumn{2}{|c|}{ Number } & \multirow{2}{*}{$\begin{array}{l}\text { M:F } \\
\text { ratio }\end{array}$} \\
\hline & Males (M) & Females (F) & \\
\hline Total & 460 & 254 & $1.8: 1$ \\
\hline HL & 82 & 26 & $3.2: 1$ \\
\hline NHL & 378 & 228 & $1.7: 1$ \\
\hline BL & 154 & 105 & $1.5: 1$ \\
\hline Histiocytic & 39 & 20 & $2: 1$ \\
\hline Lymphocytic & 166 & 86 & 2:1 \\
\hline Immunoblastic & 12 & 11 & $1: 1$ \\
\hline Plasma cell & & & \\
\hline lymphomas & 7 & 6 & 1:1 \\
\hline
\end{tabular}

$\mathrm{HL}=$ Hodgkin Lymphoma NHL $=$ Non Hodgkin Lymphoma BL = Burkitt Lymphoma

125 cases lacked information on gender and are therefore not included in the table.

Table 2 shows the distribution of the different histological types of NHL in the two periods. Lowgrade lymphomas were more frequent in the 19801989 series. In contrast, Burkitt lymphomas were diagnosed more often in 1964-1968. There was a statistically significant difference in occurrence of the histiocytic subtype $(p<0.01)$ in the two time periods and thehigh-gradelymphoblastic and immunoblastic subtypes were not reported in the 1964-1968 period. There was no statistically significant difference in the proportion of Burkitt $(\mathrm{p}=0.25)$ or lymphocytic $(\mathrm{p}=0.06)$ lymphomas in the two periods.

Looking at the Hodgkin lymphomas (Table 3), there was a statistically significant difference in the occurrence of subtypes; nodular sclerosis $(\mathrm{p}=0.02)$, lymphocyte-depleted $(\mathrm{p}<0.01)$ and lymphocyte- 
predominant $(\mathrm{p}<0.01)$ in the two time periods. The mixed cellularity type was the most common subtype of Hodgkin lymphoma in both series. The highest number of cases of all lymphomas was in the youngest age groups (up to 19 years) and the lowest number in the elderly (Figure 1). Burkitt lymphoma was most often observed in children younger than ten years (Figure 2). There was a steep decline in the number of cases after 19 years of age. The nonBurkitt lymphomas were relatively more common in the series from 1980-1989 compared to that from 1964-1968. Moreover, the non-Burkitt lymphomas had a bimodal age distribution in 1980-1989 and a unimodal age distribution in 1960-1964 (Figure 3). There was an almost uniform age distribution for HL during the two time periods (Figure 4).

Table 2

Frequency of the different NHL subtypes as diagnosed at the Department of Pathology, Makerere University in two time periods

\begin{tabular}{lcccccc}
\hline \multicolumn{7}{c}{ Frequency } \\
NHL & \multicolumn{7}{c}{ 1964-1968 } & \multicolumn{2}{c}{$1980-1989$} & P-value & $\tau^{2}$ \\
Histological subtype & No. & $(\%)$ & No. & $(\%)$ & & \\
\hline +Burkitt & 272 & 44.0 & 264 & 35.7 & 0.25 & 1.33 \\
+Histiocytic & 167 & 27.0 & 61 & 8.2 & $<0.01$ & 12.4 \\
§Lymphocytic & 134 & 21.6 & 255 & 34.5 & 0.06 & 0.73 \\
+Lymphoblastic & & $*$ & 121 & 16.4 & & \\
+Immunoblastic & & $*$ & 24 & 3.2 & & \\
+Plasma cell lymphomas & 46 & 7.4 & 15 & 2.0 & 0.08 & 2.90 \\
\hline
\end{tabular}

*=Not diagnosed in the 1964-1968 period $+=$ High grade lymphomas, $\S=$ Low grade lymphoma

Table 3

Frequency of Hodgkin lymphoma subtypes as diagnosed at the Department of Pathology, Makerere University in two time periods

\begin{tabular}{lcrcccc}
\hline \multirow{2}{*}{ HL subtype } & \multicolumn{7}{c}{ Frequency } & & \\
& No. & $(\% 64)$ & No. & (\%) & P-value & $\tau^{2}$ \\
& 15 & 11.7 & 15 & 15.2 & 0.02 & 5.26 \\
Nodular sclerosis & 25 & 19.5 & 13 & 13.1 & $<0.01$ & 14.40 \\
Lymphocyte-depleted & 23 & 18.0 & 24 & 24.2 & $<0.01$ & 11.54 \\
Lymphocyte-predominant & 65 & 50.8 & 47 & 47.5 & 0.13 & 2.18 \\
Mixed cellularity & 128 & 100 & 99 & 100 & & \\
\hline Total & & & & & & \\
\hline
\end{tabular}


Figure 1

Age group distribution for all lymphoma patients included in the study. The distribution of diagnosed lymphomas cases has not changed after the onset of the HIV/AIDS pandemic

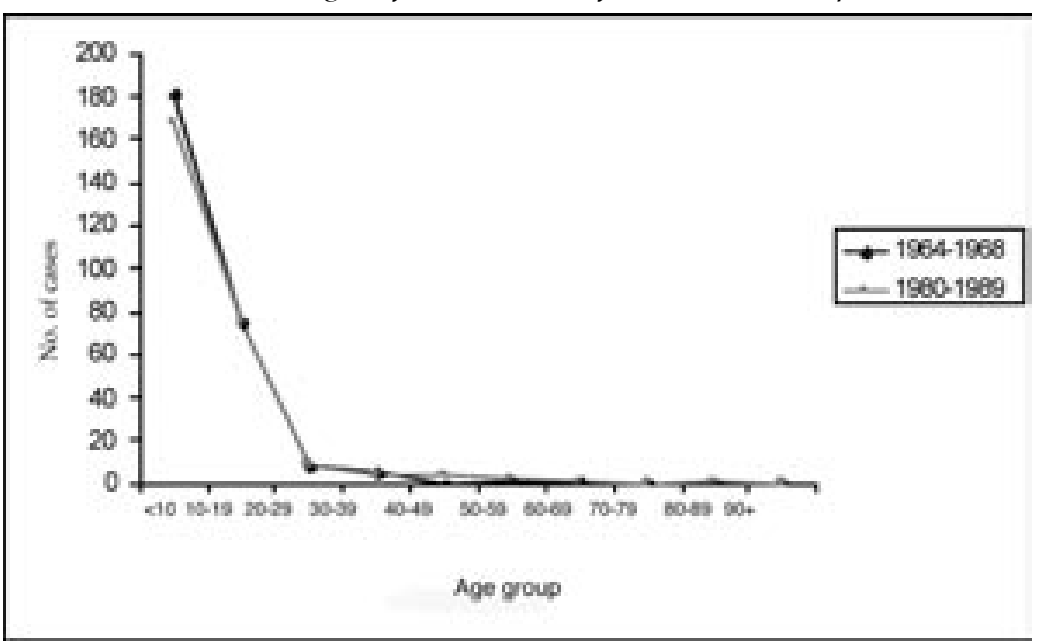

Figure 2

Age group distribution for all patients with BL diagnosed in the two time periods studied. The distribution of BL cases has not changed after the onset of the HIV/AIDS pandemic

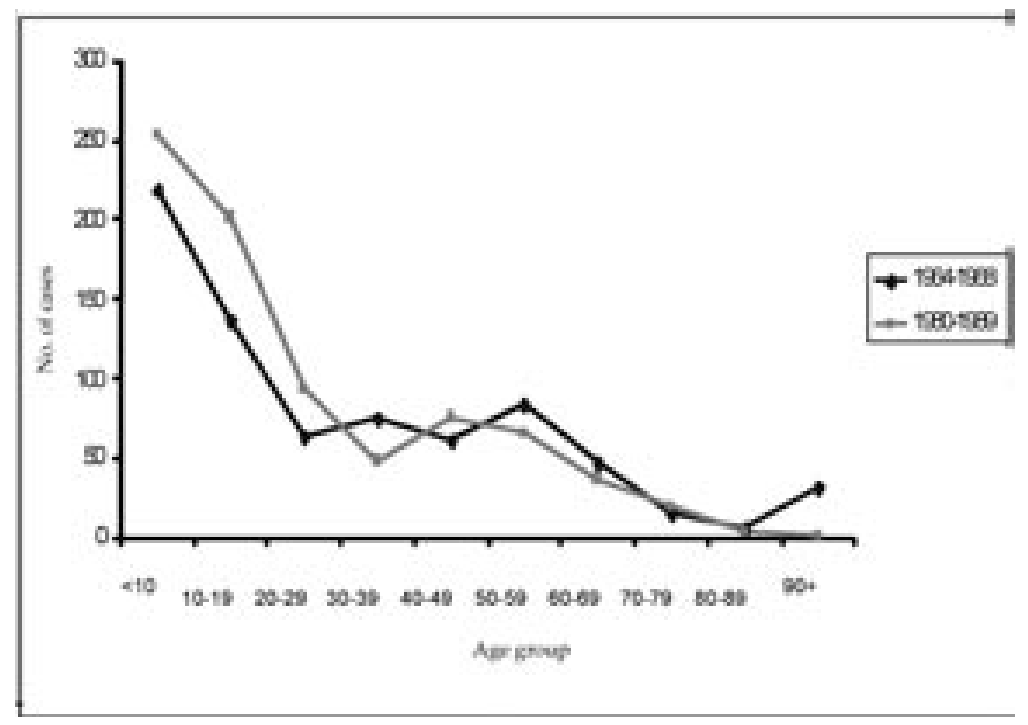

Figure 3

Age group distribution for all patients with non-Burkitt NHL in the two time periods examined. In the 1980-1989 series, the age distribution has been skewed towards the younger age groups, resulting in a bimodal distribution pattern

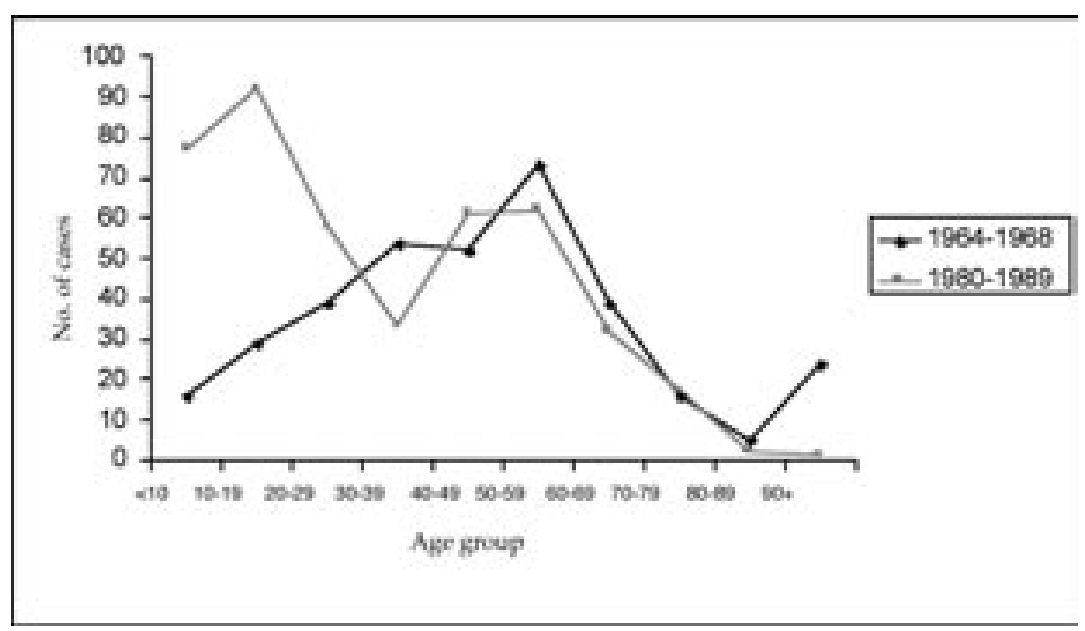


Figure 4

Age group distribution for all patients with Hodgkin lymphoma diagnosed in the two time periods examined. The distribution has not changed substantially

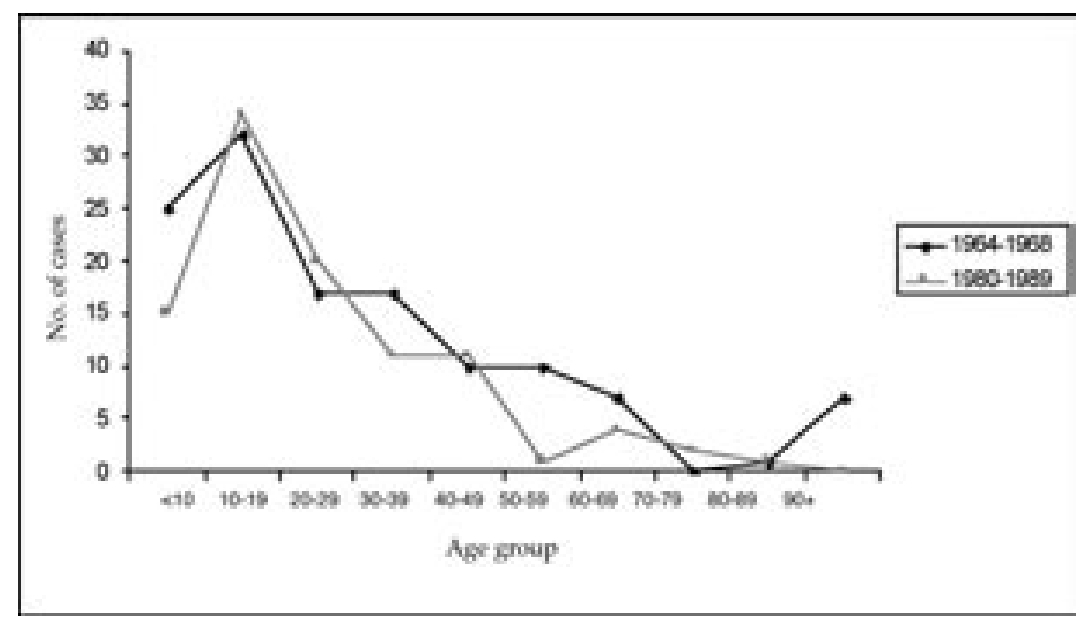

\section{DISCUSSION}

Our data show a decrease in the occurrence of histologically diagnosed lymphoma cases in the HIV / AIDS (1980-1989) period compared to the preHIV / AIDS (1964-1968) period. The male to female ratio of 1.8:1 for lymphomas diagnosed in 1980-1989 is almost similar to that found among Ugandan patients by other authors (1.67:1) but is different from the 2.5:1 found in other parts of Africa (9-11). The reduction of diagnosed lymphomas occurred despite an increase in the Ugandan population from 9.5 million in 1969 to 16.7 million in 1991. The exact cause of the decrease of diagnosed cases is not known but may be due to poorer health services as shown by the health indicators in 1989 compared to 1969 (12). This may have led to a reduced number of biopsied cases or the use of other laboratory facilities at hospitals that formerly used the University pathology laboratory. There may also have been greater reliance on clinical diagnosis and other investigative techniques like radiology. We also have considered the possibility for an actual decrease in the occurrence of lymphomas. We think this is rather unlikely. Lymphomas have been reported to be on the increase in Africa as well as in other countries partly because of HIV association with the frequency of NHL reported to be higher than that of HL (4,13-15). Previous studies have reported an association between NHL and HIV, although some studies have concluded that the incidence of NHL has remained relatively stable $(1,7,8,16)$. Our results, however, do not reflect the six-fold relative risk of developing NHL among HIV patients reported in one study in Uganda (17).

Burkitt lymphoma remains the most common lymphoma subtype diagnosed. This could indicate the aetiological factors related to this tumour are stable and that diagnostic criteria concerning this subtype have not changed. The association between HIV and BL remains confusing with some studies suggesting that Burkitt lymphoma in children is not associated with HIV infection and other studies indicating an association $(5,7,17,18)$. In our study, there were fewer cases of BL reported in the 1980s compared to the preHIV / AIDSera. Mostcases of BLoccurred in childhood and we observed no change in the age distribution of BL during the two time periods (Figure 2). This may beexplained by BLnotbeing associated withHIV or by a poor survival of children with BL and perinatal HIV. There could also be fewer cases biopsied or a change from use of biopsy to fine-needle aspiration cytology and smears for diagnostic purpose. The change in classification systems may haveled to cases previously classified as BL being categorised as new entities like thelymphoblasticlymphomas in thelastperiod. These subtypes may share morphologic features like starry sky pattern (19).

There was an increase in the proportion of the non Burkitt NHL in the 1980-1989 series as compared to the pre-AIDS era and this was noted mainly in the young age group (Table 2, Figure 3). There was a statistically significant difference in the frequency of histiocytic lymphomas in the two time periods. This is most likely due to adoption of new classification systems and terminology. Previous studies have reported that high-grade lymphomas, especially the diffuse large B-cell lymphomas (DLBCL), comprised the majority of lymphoma cases in Uganda $(18,20)$. Lymphoblastic, immunoblastic and plasmablastic lymphomas are high grade lymphomas (21). The lymphoblastic and immunoblastic subtypes contributed to $19.6 \%$ of the cases in 1980-1989 and 
are not reported in the 1964-1968 period but this may be due to change in classification systems used. The group of plasma cell lymphomas most likely includes immunoblastic, myelomas and the recent defined group of plasmablastic lymphomas. These subtypes may be difficult to distinguish morphologically (19, 22). True histiocytic lymphoma is infrequent and can imitate other high grade large cell NHL $(19,23,24)$. In both periods the diagnosis of histiocytic lymphoma has been used comprising a number of different high gradelymphoma subtypes like centroblastic, large cell anaplastic, true histiocytic and also immunoblastic in the pre-AIDS period.

Most HIV lymphomas are also reported to be high grade with two major histologic categories, diffuse large B-cell lymphoma (DLBCL) and BL (25). The boundary between BL with and without plasmacytoid differentiation is howeverindistinct and this change in morphology may be related to tumour site as well (26).The tumour typein AIDS is postulated to relate to presence of Epstein-Barr virus infection, genetic changes, degree of immune suppression and human herpes virus 8 (HHV8) infection (14). Recently lymphomas with morphologic features of immunoblastic lymphoma have been diagnosed immunophenoty pically as plasmablastic lymphoma and HIV associated $(27,28)$. There is no convincing finding in our study that high grade lymphomas with plasmablastic differentiation (plasma cell lymphoma and immunoblastic together) have increased in the HIV era.

The complete absence of reported follicular lymphomas in the 1980-1989 series was surprising. This lymphoma subtype is more often seen in the elderly patients and the lack of diagnosed cases may be explained by the young age of our patients. Other types of lymphomas not reported in our series include mycosis fungoides, central nervous system, body cavity-based and T-cell lymphomas. These lymphomas are reported to be rare or have not been documented in Africa, although some are AIDSassociated $(9,29)$. The lack of ancillary diagnostic methods most likely contributed to the lack of these subtypes.

While studies in the West reported an increased incidence of HL among patients with HIV, the situation in Uganda is not clear $(5,7,30)$. The statistically significant difference in the frequency of the subtypes during the two time periods could suggest that HIV / AIDS may have had an effect on the HL subtypes in Uganda (Table 3). Other authors have not found any association between HL and AIDS infection in Equatorial Africa (20). The change could also be partly attributed to changes in diagnostic criteria. The commonest subtype among HIV / AIDS patients in Europe is the mixed cellularity type (2, 31). We found, however, no statistically significant difference in the occurrence of the mixed cellularity subtype, hence HIV / AIDS may not have any effect on this subtype in Uganda.

Limitations: Due to poor quality of the stored slides and blocks we were unable to review the histological diagnosis. The lymphoma classification systems differed between the two periods and ancillary techniques like immunohistochemistry were not used for subtyping of lymphomas during the time periods examined.

In conclusion, Burkitt lymphoma continues to be the most common subtype of lymphoma diagnosed in Uganda. There was a statistically significant difference in the frequency of some NHL and HL subtypes as compared to the 1960s. Some lymphoma subtypes have not been reported in Uganda in the HIV / AIDS era. We recommend that it is necessary to implement up-to-date lymphoma classification systems and ancillary techniques like immunohistochemistry in Uganda.

\section{ACKNOWLEDGEMENT}

We thank the Norwegian Programme for Development Research and Education (NUFU) for funding this study.

\section{REFERENCES}

1. Dal Maso, L., Serraino, D. and Franceschi, S. Epidemiology of AIDS-related tumours in developed and developing countries. Eur. J. Cancer. 2001;37:11881201.

2. Franceschi, S., Dal Maso, L. and La Vecchia, C. Advances in the epidemiology of HIV-associated non-Hodgkin's lymphoma and other lymphoid neoplasms. Int. J. Cancer. 1999; 83: 481-485.

3. A clinical evaluation of the International Lymphoma Study Group classification of non-Hodgkin's lymphoma. The Non-Hodgkin's Lymphoma Classification Project. Blood. 1997; 89: 3909-3918.

4. Wabinga, H.R., Parkin, D.M., Wabwire-Mangen, F. and Nambooze, S. Trends in cancer incidence in Kyadondo County, Uganda, 1960-1997. Br. J. Cancer. 2000; 82:1585-1592.

5. Parkin, D.M., Wabinga, H., Nambooze, S. and Wabwire-Mangen, F. AIDS-related cancers in Africa: maturation of the epidemic in Uganda. AIDS. 1999; 13: 2563-2570.

6. Wright, D.H. Lymphoreticular neoplasms. In:Tumors in a tropical country: a survey of Uganda 1964-1968. Edited by Templeton AC. New York:Springer-Verlag; 1973. p. 271-97.

7. Mbulaiteye, S.M., Katabira, E.T., Wabinga, H., et al. Spectrum of cancers among HIV-infected persons in Africa: the Uganda AIDS-Cancer Registry Match Study. Int. J. Cancer. 2006; 118: 985-990. 
8. Wabinga, H.R., Parkin, D.M., Wabwire-Mangen, F. and Mugerwa, J.W. Cancer in Kampala, Uganda, in 1989-91: changes in incidence in the era of AIDS. Int. J. Cancer. 1993; 54: 26-36.

9. Walter, P.R., Klotz, F., Alfy-Gattas, T., et al. Malignant lymphomas in Gabon (equatorial Africa): a morphologic study of 72 cases. Hum. Pathol. 1991; 22:1040-1043.

10. Cool,C.D. and Bitter, M.A. The malignantlymphomas of Kenya: morphology, immunophenotype, and frequency of Epstein-Barr virus in 73 cases. Hum. Pathol. 1997; 28:1026-1033.

11. Tumwine, L.K., Campidelli, C., Righi, S., et al. B-cell non-Hodgkin lymphomas in Uganda: an immunohistochemical appraisal on tissue microarray. Hum. Pathol. 2008; 39: 817-823.

12. Uganda Demographic and Health Survey 1988/1989. Ministry of Health, Uganda Government; 1989.

13. Baris, D. and Zahm, S.H.Epidemiology oflymphomas. Curr. Opin. Oncol. 2000; 12: 383-394.

14. Carbone, A. and Gloghini, A. AIDS-related lymphomas: from pathogenesis to pathology. $\mathrm{Br}$. J. Haematol. 2005;130: 662-670.

15. Omoti, C.E. and Halim, N.K. Adult lymphomas in Edo state, Niger Delta region of Nigeriaclinicopathological profile of 205 cases. Clin. Lab. Haematol. 2005; 27:302-306.

16. Lucas, S.B., Diomande, M., Hounnou, A., et al. HIVassociated lymphoma in Africa: an autopsy study in Cote d'Ivoire. Int. J. Cancer. 1994; 59: 20-24.

17. Newton, R., Ziegler, J., Beral, V., et al. A case-control study of human immunodeficiency virus infection and cancer in adults and children residing in Kampala, Uganda. Int. J. Cancer. 2001; 92: 622-627.

18. Parkin, D.M., Garcia-Giannoli, H., Raphael, M. et al. Non-Hodgkin lymphoma in Uganda: a case-control study. AIDS. 2000; 14: 2929-2936.

19. Koo, C.H., Rappaport, H., Sheibani, K., et al. Imprint cytology of non-Hodgkin's lymphomas based on a study of 212 immunologically characterized cases: correlation of touch imprints with tissue sections. Hum. Pathol. 1989; 20(12 Suppl 1):1-137.

20. Lazzi, S., Ferrari, F., Nyongo, A., et al. HIV-associated malignant lymphomas in Kenya (Equatorial Africa). Hum. Pathol. 1998; 29:1285-1289.
21. Jaffe, E.S., Raffeld, M., Medeiros, L.J., et al. An overview of the classification of non-Hodgkin's lymphomas: an integration of morphological and phenotypical concepts. Cancer Res. 1992; 52(19 Suppl): 5447s5452s.

22. Andriko, J.A., Swerdlow, S.H., Aguilera, N.I. and Abbondanzo, S.L. Is lymphoplasmacyticlymphoma/ immunocytoma a distinct entity? Aclinicopathologic study of 20 cases. Am. J. Surg. Pathol. 2001; 25:742751.

23. van der Valk, P., Meijer, C.J., Willemze, R., et al. Histiocytic sarcoma (true histiocytic lymphoma): a clinicopathological study of 20 cases. Histopathology. 1984; 8(1):105-123.

24. Pileri, S., Mazza, P., Rivano, M.T., M, et al. Malignant histiocytosis (true histiocytic lymphoma) clinicopathological study of 25 cases. Histopathology. 1985; 9:905-920.

25. Gabarre, J., Raphael, M., Lepage, E., et al. Human immunodeficiency virus-related lymphoma: relation between clinical features and histologic subtypes. Am. J. Med. 2001; 111:704-711.

26. Hui, P.K., Feller, A.C. and Lennert, K. High-gradenonHodgkin'slymphoma of B-cell type. I. Histopathology. Histopathology. 1988; 12:127-143.

27. Carbone, A. Emerging pathways in the development of AIDS-related lymphomas. Lancet Oncol. 2003; 4: 22-29.

28. Hoffmann, C., Tiemann, M., Schrader, C., et al. AIDS-related B-cell lymphoma (ARL): correlation of prognosis with differentiation profiles assessed by immunophenotyping. Blood. 2005; 106:1762-1769.

29. Thomas, J.O. Acquired immunodeficiency syndromeassociated cancers in sub-Saharan Africa. Seminars Oncology. 2001; 28:198-206.

30. Biggar, R.J., Jaffe, E.S., Goedert, J.J., et al. Hodgkin lymphoma and immunodeficiency in persons with HIV / AIDS. Blood. 2006; 108: 3786-3791.

31. Frisch, M., Biggar, R. J., Engels, E. A. and Goedert, J.J. Association of cancer with AIDSrelated immunosuppression in adults. J. Am. Med. Assoc. 2001; 285:1736-1745. 